\title{
Questioni di Cuore - Appunti di cardionefrologia
}

Carissimi Amici e Colleghi,

è con piacere ed emozione che annunciamo la prossima Assemblea Costitutiva del Gruppo di Studio di Cardio-nefrologia, che si terrà presso la Sede della Società Italiana di Nefrologia di Viale dell'Università a Roma, alle 11.30 del prossimo 18 aprile.

Interverranno:

il Prof Giovambattista Capasso, Presidente eletto della SIN;

il Prof Mario Timio, vero padre putativo della Cardio-nefrologia italiana;

il Dr Moreno Malaguti, Presidente del Collegio dei Primari del Lazio.

Attendiamo tutti quelli tra Voi che vorranno partecipare, con proposte, progetti, candidature al Comitato Scientifico. Ricordiamo a tutti che quest'ultime dovranno pervenire entro e non oltre 24 ore dall'inizio dell'Assemblea, all'indirizzo mail dilullo.luca@inwind.it. Tutti i presenti dovranno essere in regola con l'iscrizione alla SIN per il 2012.

Un grazie sentito a tutti i Soci Promotori, agli amici Luca Di Lullo e Fulvio Fiorini, per l'impegno profuso nel seguire da vicino la delicata fase di gestazione del Gruppo di Studio.

A nome dei Soci Promotori

Fulvio Floccari 